Arthroskopie 2016 $29: 3-4$

DOI 10.1007/s00142-015-0058-6

Online publiziert: 25. Januar 2016

(c) Springer-Verlag Berlin Heidelberg 2015

CrossMark

\author{
P. U. Brucker ${ }^{1} \cdot$ T. Zantop ${ }^{2}$ \\ 'OrthoPlus München, München, Deutschland \\ ${ }^{2}$ Sportorthopaedicum, Straubing, Deutschland
}

\title{
Return to Sports - Was sollte der Operateur wissen?
}

Sehr geehrte Leserinnen und Leser,

in unserer täglichen klinischen Arbeit ist es unser Ziel, den Patienten unter Berücksichtigung der jeweils nationalen Rahmenbedingungen des Gesundheitssystems bestmöglich zu versorgen. Für den arthroskopisch tätigen Operateur und Gelenkchirurgen geht es hierbei darum, dass er dem Patienten einerseits eine individuelle, andererseits aber auch eine State-of-the-Art-Therapie nach den gültigen operativen Standards zukommen lässt.

Um ein optimales operatives Ergebnis zu erzielen, bedarf es neben einer präoperativ suffizienten Diagnostik und dem operativen Eingriff selbst einer adäquaten Nachbehandlung. Viele Jahre basierte die Beurteilung des postoperativen Verlaufs von ärztlicher Seite auf den klinischen Befunden im Rahmen der postoperativen Nachkontrollen, die z. T. durch bildgebende Verfahren ergänzt wurden. Die Beurteilung des klinischen Befunds nach Gelenkoperationen - unabhängig ob offen oder arthroskopisch - fokussierte dabei auf die Aspekte Reizzustand bzw. Reizfreiheit des Gelenks und der umliegenden Strukturen, aktive und passive Beweglichkeit des Gelenks, Bandund Kapselstabilität sowie Gelenk- und periartikuläre Muskelumfänge. In Übereinstimmung mit dem subjektiven Beschwerdebild des Patienten wurde bei unauffälligem subjektiven und objektiven Status der Patient wieder für sportliche Aktivitäten freigegeben. Allerdings sind diese Faktoren für die definitive Beurteilung einer Sportfähigkeit nicht ausreichend.

Unabhängig vom Lebensalter besteht in unserer Gesellschaft ein hoher An- spruch und Wunsch auf körperliche und sportliche Aktivität, was von den $\mathrm{Pa}$ tienten regelmäßig in den Anamneseund Aufklärungsgesprächen artikuliert wird. Jedem Operateur bzw. Gelenkchirurg ist bewusst, dass verschiedene sportliche Aktivitäten bzw. Sportarten und unterschiedliche Sportlevel jeweils relevante Unterschiede hinsichtlich Verletzungsrisiken und -häufigkeiten nach sich ziehen. Es gehört zu den Aufgaben des Operateurs, die operierten Sportler im postoperativen Verlauf herauszufiltern, bei denen in ihrer Sportart aufgrund individueller internaler Faktoren ein erhöhtes Risiko besteht. Dies gilt insbesondere bei ligamentären Stabilisierungsoperationen am Gelenk-Kapsel-Bandapparat, da hier das Risiko einer Re-Instabilität im Vergleich zur Primärverletzung deutlich erhöht ist.

\section{I) Der subjektive und objektive klinische Status des Patienten ist für eine Beurteilung seiner Sportfähigkeit nicht ausreichend}

Welcher Patient ist im Rahmen der Wiederaufnahme von sportlichen Aktivitäten einem Risiko auf Re-Verletzung ausgesetzt?

Diese Frage ist nicht immer einfach $\mathrm{zu}$ beantworten, da das Gesamtrisiko einer erneuten Verletzung postoperativ multifaktoriell ist und deshalb einer entsprechend differenzierten und mehrschichtigen Analyse bedarf. Dies betrifft neben dem strukturellen Gelenkstatus auch die periartikuläre Muskelfunktion hinsichtlich deren Ansteuerung, die Seitzu-Seit-Symmetrie und die muskuläre
Agonist-Antagonist-Ratio. Des Weiteren sind anthropometrische und funktionellbiomechanische Aspekte (z. B. strukturelles vs. funktionelles Achsen-Malalignment, Fehlstellungen) zu beachten. Hier ist die Datenlage von Risikoanalysen beim Kniegelenk im Vergleich zu anderen Gelenken derzeit sicherlich am weitesten fortgeschritten, aber auch an anderen Gelenken finden derartige Analysen zunehmend Berücksichtigung im wissenschaftlichen Kontext.

Inwieweit diese Risikoanalysen z. B. am Kniegelenk aktuell bei den Mitgliedern der AGA in der Klinik bzw. Praxis umgesetzt werden, ist derzeit nicht bekannt, auch vor dem Hintergrund, dass diese Analysen zum Teil zeitintensiv sind und in der Kostenerstattung der Krankenkassen nur unzureichend abgebildet werden. Eine Umfrage unter AGA-Instruktoren vor wenigen Jahren zeigte, dass die meisten der Instruktoren zeitbasiert und anhand klinischer Befunde (negativer Lachman-Test, freie Kniebeweglichkeit) eine uneingeschränkte Wiederaufnahme der sportlichen Aktivität nach vorderer Kreuzbandrekonstruktion festlegen, wohingegen einzelne Funktionstestungen (z. B. Single-Leg-Hop-Test) nur eine untergeordnete Rolle spielen [2].

Auch wenn bisher keine hohe Evidenz für die Return-to-Sport-Testung gemäß den wissenschaftlichen Kriterien [1] für viele Gelenkoperationen gegeben ist, werden funktionelle Testverfahren in den nächsten Jahren zunehmend Einzug in den klinischen Alltag finden. Wie dies im eigenen klinischen Umfeld umgesetzt werden kann, sollen die nachfolgenden Artikel in dieser Ausgabe praxisorientiert darstellen, ohne dabei den wissenschaftlichen Background außer Acht zu lassen. 
Allerdings besteht noch Nachholbedarf einer kritischen Auseinandersetzung mit diesen funktionellen Testverfahren unter wissenschaftlichen Gesichtspunkten hinsichtlich deren Evidenz.

Wir hoffen, dass wir Ihnen mit Hilfe der folgenden Beiträge nicht nur die Thematik um den „Return-to-Sport“ näherbringen können, sondern auch Ihre Patienten davon profitieren, um erfolgreich und verletzungsfrei in den Sport wieder zurückkehren zu können. Darüber hinaus sollten wir uns als Mitglieder der AGA in Zukunft auch gesundheitspolitisch dafür einsetzen, dass wissenschaftlich etablierte funktionelle Testverfahren und deren klinisch-therapeutische Konsequenzen von den zuständigen Kostenträgern auch finanziell getragen und damit fester Bestandteil der Nachbehandlung werden, um schlussendlich das klinische Endergebnis unserer operativen Eingriffe weiter zu verbessern bzw. zu optimieren.

Ihre

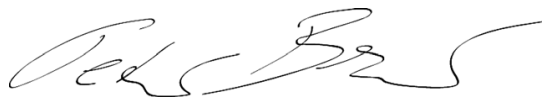

Peter Brucker

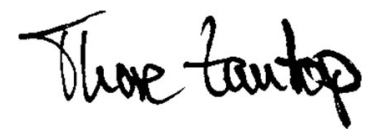

Thore Zantop

\section{Korrespondenzadresse}

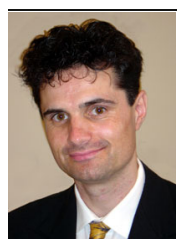

PD Dr. med. Dipl. Sportl.

P. U. Brucker

OrthoPlus München

Alte Börse, Lenbach-

platz 2a (am Stachus),

80333 München, Deutschland

p.brucker@orthoplus-muc.de

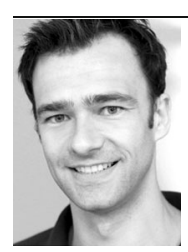

PD Dr. med. T. Zantop

Sportorthopaedicum

Bahnhofplatz 27,

94315 Straubing,

Deutschland

Interessenkonflikt. P.U. Brucker und T. Zantop geben an, dass kein Interessenkonflikt besteht.

\section{Literatur}

1. Brucker PU (2011) Evidence-based vs. Eminencebased Medicine in der Orthopädie. Arthroskopie 24:189-193

2. Petersen W, Zantop T (2013) Return to play following $A C L$ reconstruction: survey among experienced arthroscopic surgeons (AGA instructors). Arch Orthop Trauma Surg 133:969-977
Hier steht eine Anzeige.

Springer 